\title{
LINEAR MAPPINGS BETWEEN TOPOLOGICAL VECTOR SPACES
}

\author{
B. D. CRAVEN \\ (Received 3 July 1970; revised 3 February 1971) \\ Communicated by E. Strzelecki
}

\section{Introduction}

If $A$ and $B$ are locally convex topological vector spaces, and $B$ has certain additional structure, then the space $L(A, B)$ of all continuous linear mappings of $A$ into $B$ is characterized, within isomorphism, as the inductive limit of a family of spaces, whose elements are functions, or measures. The isomorphism is topological if $L(A, B)$ is given a particular topology, defined in terms of the seminorms which define the topologies of $A$ and $B$. The additional structure on $B$ enables $L(A, B)$ to be constructed, using the duals of the normed spaces obtained by giving $A$ the topology of each of its seminorms separately.

The representation theorems lead to explicit representations of $L(A, B)$, in terms of functions, or measures, depending on two variables, if $A$ and $B$ are certain function spaces. Simple proofs are obtained for some known cases - when $A$ or $B$ is $C(P)$, the space of continuous complex functions on a compact Hausdorff space $P$ (Dunford and Schwartz [4] give a representation which includes this case), and when $A=L^{P}(P)(1<p<\infty)$ (for which Cac [2] has given a representation) - but by different methods from these authors. But in addition, explicit representations, which appear to be new, are obtained for certain pairs of spaces which are not Banach spaces; when $A$ or $B$ are spaces of Schwartz distributions or test functions [7], having compact support. For example, a continuous linear mapping from Schwartz test functions into $C(P)$ may be identified with a suitable indexed family of Schwartz distributions.

\section{Calibrations and structured spaces}

If $A$ and $B$ are convex spaces (locally convex Hausdorff topological vector spaces), let $L(A, B)$ denote the space of all continuous linear mappings from $A$ into $B$. Denote by $C(W)$ the space of all bounded continuous complex functions on the Hausdorff space $W$, with the uniform norm. The topology of a convex 
space $A$ can be specified by a (non-unique) calibration, namely a set of seminorms $\left\{\|\cdot\|_{\lambda}: \lambda \in \Lambda\right\}$; similarly let $\left\{\|\cdot\|_{y}: \gamma \in \Gamma\right\}$ be a calibration for $B$.

The topology of $A$ is unchanged by adjoining to the given calibration for $A$ the maximum of each finite subset of the seminorms. The resulting calibration will be called saturated; it has the property (Bourbaki [1], page 97) that $\Lambda$ is a directed set with pre-ordering $\geqq$, where for nets $\left\{x_{\alpha}\right\}$ in $A$,

$$
\left\|x_{\alpha}\right\|_{\mu} \rightarrow 0 \text { and } \mu \geqq \lambda \Rightarrow\left\|x_{\alpha}\right\|_{\lambda} \rightarrow 0 \text {, }
$$

or equivalently

$$
\mu \geqq \lambda \Leftrightarrow \exists k=k(\lambda, \mu):\|x\|_{\lambda} \leqq k\|x\|_{\mu} \quad(\forall x \in A) .
$$

Remarks. If $\mu \geqq \lambda$ and $\lambda \geqq \mu$, then the seminorms $\|\cdot\|_{\mu}$ and $\|\cdot\|_{\lambda}$ are (topologically) equivalent.

Let $A$ be a convex space whose calibration is saturated. Denote by $A_{\lambda}$ the factor space $A / \sigma$, where $\sigma$ is the equivalence relation $x \sigma y$ iff $\|x-y\|_{\lambda}=0$, and $A_{\lambda}$ has the topology given by the corresponding quotient seminorm $\|\cdot\|_{\lambda}$. Denote by $A_{\lambda}^{\tilde{\lambda}}$ the completion of $A$, and by $A_{\lambda}^{\prime \prime}$ the dual of $A_{\lambda}^{\tilde{\lambda}}$.

Definition. A convex space $B$ will be called structured if its elements are bounded functions from a set $W$ into a Banach space $H$, and if the topology of $B$ is specified by seminorms $\|\cdot\|_{\gamma}(\gamma \in \Gamma)$ of the form

$$
\|y\|_{\gamma}=\sup _{w \in W}\left|\left(K_{\gamma} y\right)(w)\right| \quad(y \in B, \gamma \in \Gamma)
$$

where $K_{\gamma}: B \rightarrow B$ is a linear mapping (not necessarily continuous), $|\cdot|$ denotes the norm in $H$, and the set $\left\{K_{\gamma}: \gamma \in \Gamma\right\}$ includes the identity mapping, say for $\gamma=0$.

ExAmples. Let $D(I)$ denote the space of infinitely differentiable complex functions $x$, having support in the interval $I$ in Euclidean $n$-space, with topology given by either of the equivalent sets of seminorms:

$$
\begin{aligned}
& \|x\|_{\lambda}=\sup _{t \in I}\left|x^{(\lambda)}(t)\right| \\
& \|x\|_{\lambda}^{\prime}=\max _{j \leqq \lambda}\|x\|_{j} .
\end{aligned}
$$

Here $\lambda \in \Lambda_{s}$, the set of $n$-tuples $\lambda=\left(\lambda_{1}, \lambda_{2}, \cdots, \lambda_{n}\right)$ of non-negative integers, ordered by $\lambda \leqq \lambda^{\prime}$ iff $\lambda_{j} \leqq \lambda_{j}^{\prime}$ for all $j$, and $x^{(\lambda)}$ denotes the partial derivative of $x$ of order $\left(\lambda_{1}, \cdots, \lambda_{n}\right)$. Let $E(I)$ denote the space of the restrictions to $I$ of infinitely differentiable complex functions on $n$-space, with topology given by (4) or (5). Then $\boldsymbol{D}(I)$ and $\boldsymbol{E}(I)$ are structured, in terms of the calibration (4); the equivalent calibration (5) is saturated.

Any Banach space $B$ is structured, since each $y \in B$ may be represented, by its natural mapping into the second dual space $B^{\prime \prime}$, as a complex function 
on the unit sphere in $B^{\prime}$ (or, using Choquet's theorem, as a function on the set of extreme points of the unit ball in $B^{\prime}$ ); then (3) is immediate, with $K$ as the identity mapping, and $W$ the domain of the functions.

\section{Natural topology for $L(A, B)$}

Let $T \in L(A, B)$, where $A$ and $B$ are convex spaces, and the calibration of $A$ is saturated. Since $T$ is continuous, for each $\gamma \in \Gamma$ there are $\lambda_{i}, \delta^{\prime}, r$ such that

$$
\|x\|_{\lambda_{i}}<\delta^{\prime} \quad(i=1,2, \cdots, r(\gamma)) \Rightarrow\|T x\|_{\lambda}<1 .
$$

Since $\Lambda$ is a directed set, there is $\lambda \in \Lambda$ with $\lambda \geqq \lambda_{i}(i=1,2, \cdots, r)$. Then, by (1), there is $\delta$ such that

$$
\|x\|_{\lambda}<\delta \Rightarrow\|T x\|_{\gamma}<1
$$

The values of $\lambda=\lambda(\gamma, T)$ determine, for each $T \in L(A, B)$, a (non-unique) function $\Delta: \Gamma \rightarrow \Lambda$, which will be called an index function for $T$. The set $S(\Gamma, \Lambda)$ of all functions from $\Gamma$ into $\Lambda$ is partially ordered by

$$
\Delta_{\beta} \geqq \Delta_{\alpha} \Leftrightarrow \Delta_{\beta}(\gamma) \geqq \Delta_{\alpha}(\gamma) \quad(\text { all } \gamma \in \Gamma) ;
$$

denote also $\Delta_{\beta}>\Delta_{\alpha} \Leftrightarrow \Delta_{\beta} \geqq \Delta_{\alpha}$ and $\Delta_{\beta} \neq \Delta_{\alpha}$. From (1), if $\Delta$ is an index function for $T$, then so also is any $\Delta^{\prime} \geqq \Delta$. If, in particular, $A$ is countably semi-normed, then there exists a minimal (in terms of $\geqq$ ) index function for $T$; denote it by $\Delta_{\min }^{T}$.

Denote by $M(\Delta)$ the subspace of $L(A, B)$ consisting of those $T \in L(A, B)$ for which there is an index function $\leqq \Delta \in S(\Gamma, \Lambda)$. Now

$$
\Delta_{\alpha} \leqq \Delta_{\beta} \Rightarrow M\left(\Delta_{\alpha}\right) \subset M\left(\Delta_{\beta}\right)
$$

denote by $i_{\alpha \beta}$ this embedding of $M\left(\Delta_{\alpha}\right)$ in $M\left(\Delta_{\beta}\right)$.

Since $T \in L(A, B)$, each of the seminorms

$$
\|T\|_{\gamma, \mu}=\sup \left\{\|T x\|_{\gamma}:\|x\|_{\mu} \leqq 1\right\} \quad(\gamma \in \Gamma)
$$

is finite, if $\mu=\Delta(\gamma)$ for some index function $\Delta$ of $T$. Topologise $M(\Delta)$ by the seminorms $\|T\|_{\gamma, \Delta(\gamma)}(\gamma \in \Gamma)$. If $\Delta_{\alpha}$ and $\Delta_{\beta}$ are index functions of $T$, with $\Delta_{\alpha} \leqq \Delta_{\beta}$, let $\lambda=\Delta_{\alpha}(\gamma)$ and $\mu=\Delta_{\beta}(\gamma)$, for given $\gamma \in \Gamma$; since $\mu \geqq \lambda$,

with $k$ given by (2); hence

$$
\|x\|_{\lambda} \leqq k\|x\|_{\mu},
$$

therefore

$$
\left\{x:\|x\|_{\mu} \leqq 1\right\} \subset\left\{x:\|x\|_{\lambda} \leqq k\right\}
$$

$$
\|T\|_{\gamma \mu} \leqq k^{-1}\|T\|_{\gamma, \lambda} \quad(\mu \geqq \lambda) .
$$

Consequently, $i_{\alpha \beta}$ is continuous. 
Since also

$$
\Delta_{\alpha} \leqq \Delta_{\beta} \leqq \Delta_{\delta} \Rightarrow i_{\alpha \delta}=i_{\beta \delta} \circ i_{\alpha \beta},
$$

the family $\left\{M\left(\Delta_{\alpha}\right) ; i_{\alpha \beta}\right\}$ of spaces and mappings is an inductive spectrum over $S(\Gamma, \Lambda)$ (Dugundji [3], page 420). The inductive limit space of this spectrum is the quotient space $\Sigma_{\alpha} M\left(\Delta_{\alpha}\right) / \equiv$, where $\Sigma_{\alpha}$ denotes free union over $S(\Gamma, \Lambda)$ and $\equiv$ denotes the equivalence relation

$$
T_{\alpha} \in M\left(\Delta_{\alpha}\right) \equiv T_{\beta} \in M\left(\Delta_{\beta}\right)
$$

iff there exists $\delta \geqq \alpha, \beta$ such that

$$
i_{\alpha \delta} T_{\alpha}=i_{\beta \delta} T_{\beta}
$$

It will be convenient to call the topology of this' inductive limit space the natural topology for $L(A, B)$. This topology is locally convex (Robertson and Robertson [6], page 79, Prop. 4), and, for given topologies for $A$ and $B$, it is clearly independent of the particular choice of calibrations for $B$, or for $A$ so that (1) and (2) hold. If $A$ and $B$ are normed spaces, the natural topology is the operator norm topology.

The natural topology is a topology of uniform convergence; it could, of course, be expressed in terms of neighbourhoods instead of seminorms, but this does not offer any obvious simplification.

\section{Representation theorems}

Let $A$ be a convex space whose calibration is saturated; let $B$ be a convex space whose elements are functions whose domain is a set $W$. A subspace $M$ of $L(A, B)$ is represented by a vector space $Q$, whose elements are functions (or measures, or distributions) $g$ whose domain is $X \times W$ (where $X$ is a given set) if there is a bijection $\phi$ of $M$ onto $Q / \rho$, where $o$ is as equivalence relation on $Q$, and a bilinear form $F[\cdot, \cdot]$ such that

$$
(T x)(w)=F[x, g(\cdot, w)]
$$

where $x \in A, T \in M, w \in W$, and $g$ denotes a representative of the equivalence class $[g]=\phi(T) \in Q / \rho$. The equivalence relation $\rho$ will not be mentioned if it is the identity. The representation is topological if also $M$ and $Q$ are topological vector spaces, and $\phi$ maps the topology of $M$ onto that of $Q / \rho$.

As an example of (12), consider $A$ as a space of real-valued functions on a measure space $Y$, and $T$ defined by

$$
(T x)(w)=\int_{Y} x(y) g_{T}(y, w) d \mu(y)=F\left[x, g_{T}(\cdot, w)\right] .
$$

If each subspace $M\left(\Delta_{\alpha}\right)$ of $L(A, B)$ is topologically represented by a topolog- 
ical vector space $Q\left(\Delta_{\alpha}\right)$ then, since the representation is a topological isomorphism, there is a bijection $\phi^{*}$ of the inductive limit space, $M^{*}$ say, of the $M\left(\Delta_{\alpha}\right)$ onto the inductive limit space, $Q^{*}$ say, of the $Q\left(\Delta_{\alpha}\right)$; and $\phi^{*}$ maps the topology of $M^{*}$ onto that of $Q^{*}$, since $E^{*}$ does not change the values of the seminorms $\|T\|_{\gamma, \mu}$. The space $Q^{*}$ will then be called an inductive representation of $\mathrm{M}^{*}$, or of $L(A, B)$.

THEOREM 1. Let $B$ be a structured space, of functions which map $W$ into a Banach space $H$; let $B$ have calibration $\left\{\|\cdot\|_{\gamma}: \gamma \in \Gamma\right\}$. Let $A$ be any convex space, whose calibration $\left\{\|\cdot\|_{\lambda}: \lambda \in \Lambda\right\}$ is saturated. For each $\lambda \in \Lambda$, let $V_{\lambda}$ be a Banach space of functions (or complex measures, or distributions) defined on a set $X$, and $\sigma_{\lambda}$ an equivalence relation on $V_{\lambda}$, such that a congruence (an isometric isometry) between $L\left(A_{\lambda}^{\tilde{\lambda}}, H\right)$ and $V_{\lambda} / \sigma_{\lambda}$ is established by

$$
f(x)=F_{\lambda}\left[x, f^{*}\right] \text {, }
$$

where $x \in A_{\lambda}^{\tilde{\lambda}}, f \in \boldsymbol{L}\left(A_{\lambda}^{\tilde{\lambda}}, H\right), f^{*} \in V_{\lambda}$, and $F_{\lambda}$ is a bilinear form, which may depend on $\lambda$.

Then $L(A, B)$ is inductively represented by the inductive limit of a family of spaces $U^{*}(\Delta)$, where $\Delta \in S(\Gamma, \Lambda)$, and $U^{*}(\Delta)$ is a subspace of

$$
\left(V_{\Delta(0)} / \sigma_{\Delta(0)}\right) \times W \text {. }
$$

If $T \in L(A, B)$, and $\Delta$ is an index function for $T$, then

$$
\begin{aligned}
(T x)(w) & =F_{\Delta(0)}[x, g(\cdot, w)] ; \\
T_{\gamma, \Delta(\gamma)} & =\sup _{w \in W}\left\|K_{\gamma, \Delta}^{*} g(\cdot, w)\right\| ;
\end{aligned}
$$

where $x \in A, w \in W, g(\cdot, w) \in V_{\Delta(0)}$, and

$$
K_{\gamma, \Delta}^{*}: V_{\Delta(0)} \rightarrow V_{\Delta(\gamma)}
$$

is a linear mapping determined by $K_{\gamma}$. The representation is topological if $L(A, B)$ has its natural topology and $U^{*}(\Delta)$ is topologised by the seminorms $\|T\|_{\gamma, \Delta(\gamma)}(\gamma \in \Gamma)$.

Remarks. If $H=C$, the complex field, then each $f$ in the Banach space $A_{\lambda}^{\sim \prime}$ may be represented as a complex function on the unit sphere of $A_{\lambda}^{\sim}$ " (or on the set of extreme points of the unit ball in $A_{\lambda}^{\sim} "$, using Choquet's theorem.) In this sense, (13) is trivial. In various particular cases (see later theorems) $V_{\lambda}$ can be given explicitly as a space of complex functions or measures.

Not all $\Delta \in S(\Gamma, \Lambda)$ need contribute to the inductive limit.

If the $V_{\lambda}$ are function spaces then, for each $\Delta$, the subspace $M(\Delta)$ of $L(A, B)$ is isomorphic to a space of functions $W \rightarrow V_{\Delta(0)}$, for which the seminorms (15) are of the form (3); hence each subspace $M(\Delta)$ is also a structured space. 
If $A$ is countably normed, and, for each $T, \Delta_{m i n}^{T}(\gamma)$ is independent of $\gamma$, then $L(A, B)$ is inductively represented by the inductive limit of a sequence of spaces $U_{\lambda}^{*}(\lambda=0,1, \cdots)$, where $U_{\lambda}^{*}$ is a subspace of $\left(V_{\lambda} / \sigma_{\lambda}\right) \times W$. In tparticular, if $A$ is a normed space, then $L(A, B)$ is represented by a subspace $U$ of $\left(V_{0} / \sigma_{0}\right) \times W$, with the topology defined by the seminorms

$$
\|T\|_{\gamma}=\sup _{w \in W}\left\|K_{\gamma, \Delta}^{*} g(\cdot, w)\right\| \quad\left(\gamma \in \Gamma, g(\cdot, w) \in V_{0}\right) .
$$

If $A$ is a convex space with the Mackey topology (so in particular if $A$ is barrelled), then the space of all linear mappings of $A$ into $B$ which are continuous in the given topology of $A$ and the weak topology of $B$ coincides with $L(A, B)$, so is also represented by Theorem 1. For if $T$ is continuous from $A$ with strong topology to $B$ with weak topology, then $T$ is continuous from $A$ with weak topology to $B$ with weak topology ([6], page 39, Prop. 13); so if $A$ has its Mackey topology, $T$ is continuous from $A$ with strong topology to $B$ with strong topology; the converse is immediate.

Proof of Theorem 1. Let $\Delta$ be an index function for $T \in L(A, B)$; let $\gamma \in \Gamma$; let $\lambda=\Delta(\gamma)$. For fixed $\gamma$, define the linear mapping $f_{w}: A \rightarrow H$ by $f_{w}=\left(K_{\gamma} T.\right)(w)$. Since

$$
\begin{aligned}
\sup _{w \in W}\left|f_{w}(x-y)\right| & =\sup _{w \in W}\left|\left(K_{\gamma} T(x-y)\right)(w)\right| \\
& =\|T(x-y)\|_{\gamma} \\
& \leqq\|T\|_{\gamma, \lambda}\|x-y\|_{2},
\end{aligned}
$$

$f_{w}$ defines a unique element (also written $f_{w}$ ) of $L\left(A_{\lambda}, H\right)$. Since

$$
\sup _{w \in W\|x\|_{\lambda} \leqq 1}\left|f_{w}(x)\right|=\sup _{\|x\|_{\lambda} \leqq 1}\|T x\|_{\gamma}=\|T\|_{\gamma, \lambda}<\infty \text { since } \lambda=\Delta(\gamma),
$$

the mappings $f_{w}(w \in W)$ are equicontinuous on $A_{\lambda}$.

By continuity, $f_{w}$ can be extended, without increase of norm, to a continuous mapping $f_{w}^{*}: A_{\lambda}^{\tilde{\lambda}} \rightarrow H$. By (13),

$$
f_{w}^{*}(x)=F_{\lambda}\left[x, g_{\gamma, \lambda}(\cdot, w)\right] ;
$$

where $x \in A_{\lambda}^{\tilde{\lambda}}$, and $g_{\gamma, \lambda}(\cdot, w)$ is written for the function (or complex measure or distribution) $f^{*}$ corresponding to $w \in W$. Thus, for $x \in A \subset A_{\lambda}^{\tilde{\lambda}}$, and $\Delta$ any index function for $T$,

$$
\left(K_{\gamma} T x\right)(w)=F_{\Delta(\gamma)}\left[x, g_{\gamma, \Delta(\gamma)}(\cdot, w)\right] .
$$

From (18), with $\lambda=\Delta(\gamma)$,

$$
\|T\|_{\gamma, \lambda}=\sup _{w \in W}\left\|f_{w}\right\|=\sup _{w \in W}\left\|g_{\gamma, \lambda}(\cdot, w)\right\| \quad(\gamma \in \Gamma),
$$


where $\left\|g_{\gamma, \lambda}(\cdot, w)\right\|$ denotes the norm in $V_{\lambda}$, since the mapping $f \rightarrow f^{*}$ in (13) is an isometry.

Equation (20) defines a linear mapping $\psi_{\gamma}$ of $K_{\gamma} T$ onto $\left[g_{\gamma, \Delta(\gamma)}(\cdot, \cdot)\right]$, the equivalence class in

$$
\left(V_{\Delta(\gamma)} / \sigma_{\Delta(\gamma)}\right) \times W
$$

of which $g_{\gamma, \Delta(\gamma)}(\cdot, \cdot)$ is a representative. Since $F_{\Delta(\gamma)}$ is a bilinear form, and the mapping $f \rightarrow\left[f^{*}\right]$ defined by (13) is a bijection, $\psi_{\gamma}$ has zero kernel, so $\psi_{\gamma}^{-1}$ exists. Denote by $\sigma^{*}$ the canonical mapping of $V_{\Delta(0)}$ into

$$
V_{\Delta(0)} / \sigma_{\Delta(0)}
$$

denote by $e_{\gamma}$ any linear embedding of $V_{\Delta(\gamma)} / \sigma_{\Delta(\gamma)}$ into $V_{\Delta(\gamma)}$. Define

by

$$
K_{\gamma, \Delta}^{*}: V_{\Delta(0)} \rightarrow V_{\Delta(\gamma)}
$$

$$
K_{\gamma, \Delta}^{*}=e_{\gamma} \circ \psi_{\gamma} \circ K_{\gamma} \psi_{0}^{-1} \circ \sigma^{*} .
$$

Then $K_{\gamma, \Delta}^{*}$ maps $g_{0, \Delta(0)}(\cdot, \cdot)$ onto $g_{\gamma, \Delta(\gamma)}(\cdot, \cdot)$. This, with (20), proves (14), writing $g$ for $g_{0, \Delta(0)}$.

Denote by $Z\left(\Delta_{\alpha}\right)$ the subspace of $V_{\Delta_{\alpha}(0)} \times W$ consisting of those functions $g_{0, \Delta_{\alpha}(0)}(\cdot, \cdot)$ for which all the seminorms $(21)$ are finite, with the convex topology determined by these seminorms. Since these seminorms are finite for each $T \in \boldsymbol{L}(A, B)$ for which $\Delta_{\alpha}$ is an index function, there is, by (20), a linear injection

$$
j_{\alpha \beta}: M\left(\Delta_{\alpha}\right) \rightarrow Z\left(\Delta_{\beta}\right)
$$

for each $\Delta_{\alpha}$ and $\Delta_{\beta} \geqq \Delta_{\alpha}$ in $S(\Gamma, \Lambda)$. Let $U\left(\Delta_{\alpha}\right)=j_{\alpha \alpha} M\left(\Delta_{\alpha}\right)$, with the relative topology of $Z\left(\Delta_{\alpha}\right) ; U\left(\Delta_{\alpha}\right)$ is, in general, a proper subspace of $Z\left(\Delta_{\alpha}\right)$, since the finiteness of all the seminorms (15) does not imply that $T x \in B$ for all $x \in A$.

Since $j_{\alpha \alpha}$ is a bijection onto $U\left(\Delta_{\alpha}\right)$, there is a linear injection $\phi_{\alpha \beta}=j_{\alpha \beta} \circ j_{\alpha \alpha}^{-1}$ : $U\left(\Delta_{\alpha}\right) \rightarrow Z\left(\Delta_{\beta}\right)$ which, by (11), satisfies $\phi_{\alpha \delta}=\phi_{\beta \delta} \circ \phi_{\alpha \beta}$ whenever $\Delta_{\alpha} \leqq \Delta_{\beta} \leqq \phi_{\Delta}$. Since $j_{\alpha \alpha}$ does not change the seminorms (15), $j_{\alpha \alpha}$ is continuous. Since $j_{\alpha \beta}=j_{\beta \beta} \circ i_{\alpha \beta}$ and $i_{\alpha \beta}$ is continuous, $j_{\alpha \beta}$ is a continuous mapping onto $U\left(\Delta_{\beta}\right)$; hence $\phi_{\alpha \beta}: U\left(\Delta_{\alpha}\right) \rightarrow U\left(\Delta_{\beta}\right)$ is continuous. Therefore the family $\left\{U^{*}\left(\Delta_{\alpha}\right) ; \phi_{\alpha \beta}\right\}$, where $U^{*}\left(\Delta_{\alpha}\right)=U\left(\Delta_{\alpha}\right) / \sigma_{\Delta_{\alpha}(0)}$ is an inductive spectrum over $S(\Gamma, \Lambda)$. From (15) and the definition of natural topology for $L(A, B), L(A, B)$ is inductively represented by the inductive limit of this spectrum.

THEOREM 2. Let the spaces $A$ and $B$ satisfy the hypotheses of Theorem 1; let $\Delta \in S(\Gamma, \Lambda)$; define the mapping $T: A \rightarrow B$ by $(14)$, where $g(\cdot, w) \in V_{\Delta(0)}$, $w \in W$. Let $g$ be such that $T x \in B$ whenever $x \in A$. For each $\gamma \in \Gamma$, assume that

$$
\left(K_{y} T x\right)(w)=F_{\Delta(\gamma)}\left[x, K_{\gamma, \Delta}^{*} g(\cdot, w)\right],
$$

where $K_{\gamma, \Delta}^{*}: V_{\Delta(0)} \rightarrow V_{\Delta(\gamma)}$ is a linear mapping satisfying 


$$
\sup _{w \in W}\left\|K_{\gamma, \Delta}^{*} g(\cdot, w)\right\|<\infty .
$$

Then $T \in L(A, B)$, and $\Delta$ is an index function for $T$.

Proof. Since $T$ maps $A$ linearly into $B$, it suffices to show that $T$ is continuous. From (9) and (3),

$$
\begin{array}{rlrl}
\|T\|_{\gamma, \Delta(\gamma)} & =\sup _{\|x\|_{\Delta(\gamma) \leqq 1}} \sup _{w \in W}\left|\left(K_{\gamma} T x\right)(w)\right| & \\
= & \sup _{w \in W} \sup _{\|x\|_{\Delta(\gamma) \leqq 1}\left|F_{\Delta(\gamma)}\left[x, K_{\gamma, \Delta}^{*} g(\cdot, w)\right]\right|, \text { by }(23)} \\
= & \sup _{w \in W}\left\|K_{\gamma, \Delta}^{*} g(\cdot, w)\right\| & \text { by (13) } \\
& <\infty & \text { by (24). }
\end{array}
$$

\section{Representations of particular spaces}

Let $A$ and $B$ satisfy the hypotheses of Theorem 1; define $T$ by (14). Suppose that (i) $A$ is such that $V_{\lambda}$ and $F_{\lambda}$ are known explicitly, and (ii) the subspace $U^{*}(\Delta)$ of

$$
\left(V_{\Delta(0)} / \sigma_{\Delta(0)}\right) \times W
$$

for which $T$ maps $A$ onto $B$ (rather than onto a superspace of $B$ ) can be characterized. Then the representation of $L(A, B)$ can be given explicitly. Theorems 3 to 7 give examples; in them, all functions (unless stated otherwise) are complexvalued, $I$ and $J$ are compact real intervals, $P$ and $Q$ are compact Hausdorff spaces, and $V$ denotes total variation (of a measure). If $\sigma_{\lambda}$ is not mentioned, it is the identity.

THEOREM 3. $L(C(P), C(Q))$ is isometric and isomorphic to a space of finite Radon measures $g(\cdot, w)$ on $P$, where $w \in Q$, such that $g(\cdot, w)$ is weak*-continuous in $w \in Q$, and $\sup _{w e Q} \operatorname{Vg}(\cdot, w)$ is finite. Then $T \in L(A, B)$ if and only if

$$
\begin{aligned}
(T x)(w) & =\int_{P} x(v) d g(v, w) \quad(x \in C(P), w \in Q) \\
\|T\| & =\sup _{w \in Q} V g(\cdot, w)
\end{aligned}
$$

Proof. In Theorem 1, set $A=C(P), B=C(Q) ; A^{\prime}=L\left(A_{\lambda}^{\tilde{\lambda}}, C\right)$, where $\|\cdot\|_{\lambda}$ is the uniform norm, is congruent to the space $V$ of finite Radon measures on $P$, and

$$
f(x)=F\left[x, f^{*}\right]=\int_{P} x d f^{*} .
$$

So (14) and (15) give (25) and (26), with (26) finite; and the requirement that $T$ maps into $C(Q)$ is that $g$ satisfies 


$$
\lim _{w \rightarrow w_{0}} \int_{P} x(\cdot) d g(\cdot, w)=\int_{P} x(\cdot) d g\left(\cdot, w_{0}\right)
$$

$\left(w, w_{0} \in Q\right)$, i.e. the weak*-continuity of $g(\cdot, w)$ in $w$. Conversely, if $T$ is defined by (25), and (26) and (27) hold, then $T \in L(A, B)$ by Theorem 2, since by (27), $T$ maps into $C(Q)$.

THEOREM 4. If $1<p<\infty$ and $\mu$ is a measure on $P$, then $L\left(L_{\mu}^{p}(P), C(Q)\right)$ is isomorphic and isometric to a space of functions $g(v \cdot w)(v \in P, w \in Q)$ defined by the properties:

$$
\sup _{w \in Q}\|g(\cdot, w)\|_{\Psi}<\infty\left(p^{-1}+q^{-1}=1 ;\|\cdot\|_{q} \text { is the } L_{\mu}^{q}(P) \text {-norm }\right)
$$

(29) $\int_{E} g(\cdot, w) d \mu(\cdot)$ is continuous in $w \in Q$, for each measurable subset $E \subset P$.

Then $T \in L\left(L_{\mu}^{P}(P), C(Q)\right)$ iff

$$
(T x)(w)=\int_{P} x(v) g(v, w) d \mu(v) \quad\left(x \in L_{\mu}^{p}(P), w \in Q\right)
$$

and $\|T\|$ is given by the left side of the inequality (28).

Proof. In Theorem 1, set $A=L_{\mu}^{p}(P), B=C(Q) ; A^{\prime}=L\left(A_{\lambda}^{\tilde{\lambda}}, C\right)$ is congruent to $L_{\mu}^{q}(P)$, with $F\left[x, f^{*}\right]=\int_{P} x(v) f^{*}(v) d \mu(v)(x \in A)$. So $L(A, B)$ is congruent to a subspace of $L_{\mu}^{q}(P) \times Q$, and (14) and (15) give (30) and (28); and (29) follows on substituting the characteristic function of $E$ for $x(\cdot)$ in (30), and requiring that $T x \in C(Q)$.

Conversely it suffices, by Theorem 2, to show that (28), (29) and (30) imply $T x \in C(Q)$ if $L_{\mu}^{p}(P)$. There is a simple function $\bar{x}$ such that $\|x-\bar{x}\|_{p}<\varepsilon /(4 k)$, where $k$ is the supremum in (28). Let $h(v, w)=g(v, w)-g\left(v, w_{0}\right)$, where $w, w_{0} \in Q$. Since $\bar{x}$ is a simple function, (29) requires that $\left|\int_{p} \bar{x} h d \mu\right|<\varepsilon / 2$ if $w \in N\left(w_{0}\right)$, a suitable neighbourhood of $w_{0}$, depending on $\varepsilon$. Then

$$
\begin{aligned}
\left|(T x)(w)-(T x)\left(w_{0}\right)\right| & =\left|\int_{P}(x-\bar{x}) h d \mu+\int_{P} \bar{x} h d \mu\right| \\
& \leqq\|(x-\bar{x})\|_{p}\|h\|_{q}+\left|\int_{P} \bar{x} h d \mu\right| \\
& <\varepsilon /(4 k) \cdot 2 k+\varepsilon / 2 .
\end{aligned}
$$

So $T \in C(Q)$.

THeOrem 5. If $1<p<\infty$ and $\mu$ is a measure on $P$, then $L\left(L_{\mu}^{p}(P), E(J)\right)$ is inductively represented by a space of functions $g_{r}(v, w)(v \in P ; w \in J ; r=0,1,2 \cdots)$ having the properties: 


$$
\begin{gathered}
\sup _{w \in J}\left\|g_{r}(\cdot, w)\right\|_{q}<\infty \quad\left(p^{-1}+q^{-1}=1 ;\|\cdot\|_{q} \text { is the } L_{\mu}^{q}(P) \text {-norm }\right) \\
{\left[g_{r}(\cdot, w)-g_{r}\left(\cdot, w_{0}\right)\right] /\left[w-w_{0}\right] \rightarrow g_{r+1}\left(\cdot, w_{0}\right)}
\end{gathered}
$$

in the weak $L_{\mu}^{p}$ topology on $P$, as $w \rightarrow w_{0}$. $:\left(w, w_{0} \in J\right)$. Then $T \in \boldsymbol{L}\left(L_{\mu}^{p}(P)\right.$, $E(J))$ iff $\left(\right.$ for $\left.x \in L_{\mu}^{p}(P) ; w \in J ; r=0,1,2, \cdots\right)$

$$
\left(D^{r} T x\right)(w)=\int_{P} x(v) g_{r}(v, w) d \mu(v),
$$

where $D$ is the derivative operator.

REMARKs. The seminorms $\|T\|$ (see (16)) equal the expressions on the left of $(31)$, for $r=0,1, \cdots$. The Theorem remains true for $J$ replaced by $(-\infty, \infty)$.

Proof. Let $T \in L\left(L_{\mu}^{p}(P), E(J)\right)$. For $r=0,1,2, \cdots$, the map

$$
D: E(J) \rightarrow E(J)
$$

is continuous; since also $E(J) \subset C(J)$,

$$
D^{r}(T) \in L\left(L_{\mu}^{p}(P), C(J)\right) .
$$

So (31) and (33) follow from (28) and (30) of Theorem 5. From (33), if $x \in L_{\mu}^{p}(P)$,

$$
\frac{\left(D^{r} T x\right)(w)-\left(D^{r} T x\right)\left(w_{0}\right)}{w-w_{0}}=\int_{P} x(v)\left[\frac{g_{r}(v, w)-g_{r}\left(v, w_{0}\right)}{w-w_{0}}\right] d \mu(v)
$$

Since $T x \in E(J)$, the left side of $(34) \rightarrow\left(D^{r+1} T x\right)\left(w_{0}\right)$ as $w \rightarrow w_{0}$; and (32) follows, using (33). From (3) with $K_{r}=D^{r}$, (16), and (31), the natural topology for $L\left(L_{\mu}^{p}(P), E(J)\right)$ is that given by the sequence of seminorms $\|T\|_{r}$ given by the expressions in (31).

Conversely, define $T$ by (33) with $r=0$, and assume (31) and (32); by Theorem 2 , it is required only to verify that $T x \in E(J)$ if $x \in L_{\mu}^{p}(P)$. If (33) holds for some $r \geqq 0$, then so does (34); by (32),

$$
\text { the right side of }(34) \rightarrow \int_{P} x(v) g_{r+1}\left(v, w_{0}\right) d \mu(v)
$$

as $w \rightarrow w_{0}$ in $J$; hence so does the left side; so (33) holds for $r+1$, and, by induction, for all $r$; so $T x$ is infinitely differentiable. Now

$$
\begin{aligned}
& \|T\|_{r}=\sup _{w \in J} \sup _{\|x\|_{r} \leqq 1}\left|\left(D^{r} T x\right)(w)\right| \\
& \sup _{w \in J} \sup _{\|x\|_{r} \leqq 1}\|x\| \cdot\left\|g_{r}(\cdot, w)\right\| \text { by (33) } \\
& \sup _{w \in J} \| g_{r}(\cdot, w) \\
& <\infty
\end{aligned}
$$


So $T x \in E(J)$.

Remarks. Let $P$ be a compact convex subset of Euclidean $n$-space: let $A=E(P)$ (see (4) and (5)); let $f \in A^{\prime}$. Then (compare (6)) there is a seminorm $\|\cdot\|_{\lambda}$ of $A$ such that $f$ is continuous in $\|\cdot\|_{\lambda}$. So $f$ extends by continuity to a continuous linear functional on $A_{\lambda}^{\sim}$. To each $x \in A$, attach (uniquely) the set of functions $\left\{x^{(q)}: q \leqq \lambda\right\}$; this defines an injection $j$ of $A_{\lambda}$ into the direct sum $S_{\lambda}$ of finitely many $(s$, say) copies of $C(P)$. Norm $S$ by

$$
\max _{q \leqq \lambda} \sup _{t \in P}\left|x^{(q)}(t)\right| \text {. }
$$

Then $f^{\sim}(y)=f\left(j^{-1} y\right)\left(y \in j A_{\lambda}^{\sim}\right)$ determines a functional $f^{\sim}$ on $j A_{\lambda}^{\sim}$ with the same norm, $p(f)$ say, that $f$ has as an element of $A_{\lambda}^{\sim \prime}$. The Hahn-Banach theorem extends $f^{\sim}$ to a continuous linear functional on $S_{\lambda}$, with the same norm. Then by the Riesz representation theorem, there is a (complex) measure on $P^{s}$, represented by measures $f_{q}^{*}$ on $P$, corresponding to the direct summands of $S_{\lambda}$, such that

$$
\begin{aligned}
& f(x)=\sum_{q \leqq \lambda} \int_{P} x^{(q)}(v) d f_{q}^{*}(v) \\
& p(f)=\sum_{q \leqq \lambda} V f_{q}^{*} .
\end{aligned}
$$

This proof is adapted from the representation [5] for Schwartz distributions with compact support $P$. If $f$ is such a distribution, then it is well known that

$$
f(x)=\sum_{q \leqq \lambda^{\prime}} \int_{N} x^{(q)}(v) d \int_{q}^{*}(v) \quad(x \in E(I))
$$

where $N$ is an arbitrary neighbourhood of $P$, and $I$ is an interval of $\boldsymbol{R}^{n}$, containing $P$; here the measures $f_{q}^{*}$ depend, in general, on the choice of $N$. However, if $P$ is compact convex then Schwartz [7] shows that $N$ may be replaced by $P$ in (37), provided $\lambda^{\prime}$ is replaced by $\lambda$, where $\lambda / \lambda^{\prime}$ depends on $P$ but not on $f$.

It follows that, within a topological isomorphism, $E(P)^{\prime}$ is the space of Schwartz distributions with support in $P$, and $f \in \mathrm{P}(E)^{\prime}$ iff $f$ has a representation (35), (36), for some $\lambda \in \Lambda_{s}$. It is convenient to identify $f$ with the vector $\left\{f_{r}^{*}: r \leqq \lambda\right\}$ of measures.

THEOREM 6. Let $P$ be a compact convex subset of Euclidean $n$-space; let $Q$ be a compact Hausdorff space. Then $L(E(P), C(Q))$ is topologically represented by a space of elements $g(\cdot, \cdot)$, where for each $w \in Q$,

$$
g(\cdot, w)=\left\{g_{r}(\cdot, w): r \leqq \lambda\right\}
$$

is a Schwartz distribution with support in $P$. If $T \in L(E(P), C(Q))$ and $\Delta$ is the minimal index function for $T$, then for $\lambda=\Delta(0), w \in Q$, 


$$
\begin{gathered}
(T x)(w)=\sum_{r \leqq \lambda} \int_{P} x^{(r)}(v) d g_{r}(v, w) \quad(x \in E(P)) ; \\
\|T\|_{0, \Delta(0)}=\sup _{w \in Q} p(g(\cdot, w)),
\end{gathered}
$$

where $p(\cdot)$ is defined in (36);

$$
(g \cdot, w) \text { is weak *-continuous in } w \in Q \text { with respect to } \boldsymbol{E}(P) \text {. }
$$

Conversely, if $T$ is defined by (38), and (39) and (40) hold, then $T \in L(E(P), C(Q))$.

RemarK. (40) means that, if $\langle\cdot, \cdot\rangle$ denotes evaluation of a distribution, then for each $x \in E(P)$,

$$
\langle g(\cdot, w), x(\cdot)\rangle
$$

is continuous in $w \in Q$.

Proof. Set $A=E(P), \Lambda=\Lambda_{s}$, and $B=C(Q)$ in Theorem 1: if $x \in E(P)$ and $f \in A_{\lambda}^{\sim \prime}$, then (35) and (36) hold; therefore (38) and (39) follow from Theorem $1 ;(40)$ is precisely the condition that $T$ maps into $C(Q)$. The converse is immediate from Theorem 2 .

TheOREM 7. Let $P$ and $Q$ satisfy the conditions for $P$ as in Theorem 6. Then $\boldsymbol{L}(\boldsymbol{E}(P), \boldsymbol{E}(Q))$ is topologically represented by a space of sequences

$$
\left\{g_{\gamma}(\cdot, \cdot): \gamma \in \Lambda_{s}\right\},
$$

where for each $w \in Q, g_{\gamma}(\cdot, w)$ is a Schwartz distribution with support in $P$. If

$$
T \in L(E(P), E(Q))
$$

and $\Delta$ is the minimal index function for $T$, then for $\lambda=\Delta(0), x \in E(P), w \in Q$, $\gamma \in \Lambda_{s}$,

$$
\left(D^{\gamma} T x\right)(w)=\sum_{r \leqq \lambda} \int_{P} x^{(r)}(v) d g_{\gamma, r}(v, w)=\left\langle g_{\gamma}(\cdot, w), x(\cdot)\right\rangle
$$

where

$$
\begin{aligned}
& g_{\gamma}(\cdot, \cdot)=\left\{g_{\gamma, r}(\cdot, \cdot): r \leqq \lambda\right\} ; \\
&\|T\|_{\gamma, \Delta(\gamma)}=\sup _{w \in Q} p\left(g_{\gamma}(\cdot, w)<\infty ;\right.
\end{aligned}
$$

(43) $\quad g_{\gamma}(\cdot, w)$ is weak-*-continuous in $w \in Q$ with respect to $E(P)$;

(44) $\quad \mathrm{g}_{\gamma+1}(\cdot, w)=(\partial / \partial w) g_{\gamma}(\cdot, w)$, the derivative taken in the weak-* sense on $E(P)$.

Conversely, if $T$ is defined by (41) with $\gamma=0$, and the $g_{\gamma}$ satisfy (42), (43), (44), and $\lambda \in \Lambda_{s}$, then $T \in L(E(P), E(Q))$. 
REMARK. If $w$ has components $w_{j}$, then $\partial g / \partial w$ means the vector with components $\partial g / \partial w_{j}$; and $h\left(w, w_{0}\right) /\left(w-w_{0}\right)$ means the vector with components $h\left(w, w_{0}\right) /\left(w-w_{0}\right)_{j}$.

Proof. If $T \in L(E(P), E(Q))$, and $\gamma \in \Lambda_{s}$, then $D^{\gamma} T \in \boldsymbol{L}(\boldsymbol{E}(P), C(Q)$ ) (where $D$ is the differentiation operator). Hence Theorem 6 applies, and (38), (39), (40) prove (41) (for each $\gamma$ ), (42), (43): except that $\lambda$ may depend on $\gamma$ as well as on $T$. From (41), for $w, w_{0} \in Q$,

$$
\frac{\left(D^{\gamma} T x\right)(w)-\left(D^{\gamma} T x\right)\left(w_{0}\right)}{w-w_{0}}=\frac{\left\langle g_{\gamma}(\cdot, w), x(\cdot)\right\rangle-\left\langle g_{\gamma}\left(\cdot, w_{0}\right), x(\cdot)\right\rangle}{w-w_{0}}
$$

Since $T$ maps into $E(Q),\left(D^{\gamma} T x\right)\left(w_{0}\right)$ exists, so the left side of (45) converges to it as $w \rightarrow w_{0}$, hence so does the right side. Let $\left\{w_{n}\right\} \rightarrow w_{0}$ in $Q$; let (41) hold for given $\gamma$ and $\lambda$; then

$$
\phi_{n}=\left(g_{\gamma}\left(\cdot, w_{n}\right)-g_{\gamma}\left(\cdot, w_{0}\right)\right) /\left(w_{n}-w_{0}\right)
$$

is a continuous linear mapping from $A_{\lambda}$ (where $A=\boldsymbol{E}(P)$ ), convergent as $w \rightarrow w_{0}$ to $g_{\gamma+1}\left(\cdot, w_{0}\right)$; by the uniform boundedness principle, $g_{\gamma+1}\left(\cdot, w_{0}\right)$ is also continuous on $A_{\lambda}$; hence $\lambda$ is independent of $\gamma$, and (44) holds. The converse is proved as in Theorem 6 .

COROLlary. The space $L\left(E(Q)^{\prime}, E(P)^{\prime}\right)$ of all continuous linear mappings from Schwartz distributions with support in $Q$ to Schwartz distributions with support in $P$, where $P$ and $Q$ satisfy the hypotheses of Theorem 7, has the following representation. Let

$$
U \in \boldsymbol{L}\left(\boldsymbol{E}(Q)^{\prime}, \boldsymbol{E}(P)^{\prime}\right)
$$

let $x \in \boldsymbol{E}(P)$; let $f \in \boldsymbol{E}(Q)^{\prime} ;$ by (37), $f$ may be specified in terms of (complex) measures $h_{q}$ on $Q$ by

$$
f(y)=\sum_{q \leqq \mu} \int_{Q} y^{(q)}(w) d h_{q}(w) \quad(y \in \boldsymbol{E}(Q)) .
$$

Then

$$
(U f)(x)=\sum_{r \leqq \lambda} \int_{P} x^{(r)}(v) d_{v}\left[\int_{Q} \sum_{q \leqq \mu} g_{q, r}(v, w) d h_{q}(w)\right]
$$

where the measures $g_{q, r}(\cdot, w)$ satisfy (42), (43), (44). And conversely, if $U$ is defined by (47) then $U \in L\left(E(Q)^{\prime}, E(P)^{\prime}\right)$.

Proof. Since $\boldsymbol{E}(P)$ and $E(Q)$ are reflexive metrisable convex spaces,

$$
U \in \boldsymbol{L}\left(\boldsymbol{E}(Q)^{\prime}, \boldsymbol{E}(P)^{\prime}\right)
$$

iff $U$ is the adjoint of an element $T \in L(E(P), E(Q))$; and 


$$
(U f)(x)=f(T x)
$$

Then (47) follows from (41).

\section{Acknowledgement}

I am indebted to a referee for various details which have improved the presentation of this paper.

\section{References}

[1] N. Bourbaki, Espaces vectoriels topologiques, Ch. I \& II (1st. edn.).

[2] N. P. Cac, 'Linear Transformations on some functional spaces,' Proc. Lond. Math. Soc. (3) 16 (1966), 705-776.

[3] J. Dugundji, Topology (Allyn \& Bacon, Boston, 1967).

[4] N. Dunford and J. T. Schwartz, Linear Operators, Part 1. (Interscience, New York, 1958).

[5] I. M. Gelfand and G. E. Shilov, Generalized Functions, vol. 2 (Dunod, Paris, 1964).

[6] A. P. Robertson and W. Robertson, Topological Vector Spaces (Cambridge University Press, 1964).

[7] L. Schwartz, Théorie des distributions (Hermann, Paris, new edition 1966).

Department of Mathematics

Melbourne University

Victoria, 3052

Australia 